\title{
Foreign Gene Transfer to Cardiomyocyte Using a Replication-Defective Recombinant Coxsackievirus B3 without Cytotoxicity
}

\author{
Byung-Kwan Lim Soo-Hyeon Yun Chae-Ok Gil Eun-Seon Ju Jin-Oh Choi \\ Duk-Kyung Kim Eun-Seok Jeon \\ Division of Cardiology, Samsung Medical Center, Sungkyunkwan University School of Medicine, Seoul, Korea
}

\section{Key Words}

Replication-defective recombinant coxsackievirus •

Transfer vector $\cdot$ Cardiac myocytes $\cdot$ Cardiotropic virus .

Cytotoxicity

\begin{abstract}
Background: Replication-competent coxsackievirus B3 (CVB3) has been used as a gene transfer vector for cultured cardiomyocytes and hearts in vivo. However, CVB3 induces cell lysis when it replicates in infected cells. In this study, we investigated whether a replication-defective rCVB3 vector could be generated and used as a noncytotoxic gene transfer vector for cardiomyocytes. Methods: We generated a replication-defective luciferase-expressing CVB3 plasmid. This recombinant CDNA and pCMV-P1 plasmids were amplified and cotransfected into Hek293 cells using transfection reagents. Replication-defective rLuCVB3 virus was recovered from the cells and cell culture supernatants for 3 days after transfection. The generated rLuCVB3 viruses were concentrated on a $30 \%$ sucrose cushion and semiquantified using a luciferase assay. In addition, foreign gene delivery by the rLuCVB3 was tested in cultured cardiomyocytes and intact mouse hearts after rLuCVB3 infection. Results: Luciferase was expressed in Hek293, HeLa cells and cardiomyocytes after rLuCVB3 infection. In addition, these cells did not show a significant cytopathic effect after $72 \mathrm{~h}$. Luciferase protein
\end{abstract}

expression or activity were detected for 3 days in the myocardium of rLuCVB3-infected mouse hearts without producing cytotoxicity or inflammation. Conclusion: As a proof-ofconcept, these data indicate that a replication-defective rCVB3 vector can be generated and used as a novel gene transfer system to transfect exogenous genes into cardiomyocytes without generating cytotoxicity.

Copyright $\odot 2011$ S. Karger AG, Basel

\section{Introduction}

Coxsackievirus B3 (CVB3) is a member of the picornaviridae family of enteroviruses. It has a $7.4-\mathrm{kb}$ positive single-strand RNA genome that is translated as a monocistronic polyprotein $[1,2]$. The CVB3 genome encodes capsid proteins (P1: VP4, VP2, VP3 and VP1) and nonstructural proteins $(2 \mathrm{~A}, 2 \mathrm{~B}, 2 \mathrm{C}, 3 \mathrm{~A}, 3 \mathrm{~B}, 3 \mathrm{C}$ and $3 \mathrm{D})$ that are involved in viral replication [3]. Nonstructural proteins $2 \mathrm{~A}$ and $3 \mathrm{C}$ are cotranslationally cleaved into mature peptides. CVB3 is a highly cardiotropic virus and the most common pathogen that causes human viral myocarditis. CVB3 requires the coxsackie and adenovirus receptor (CAR) to attach and penetrate the target host cells.

\section{B.-K.L. and S.-H.Y. contributed equally to this project.}

\section{KARGER}

Fax +4161306 1234

E-Mail karger@karger.ch

www.karger.com
(ㄷ) 2011 S. Karger AG, Basel

0300-5526/12/0553-0201\$38.00/0

Accessible online at:

www.karger.com/int
Eun-Seok Jeon, MD, PhD

Division of Cardiology, Samsung Medical Center

Sungkyunkwan University School of Medicine

50 Irwon-Dong, Gangnam-Gu, Seoul 135-710 (Korea)

Tel. +82 234103448 ,E-Mail esjeon@ @kku.edu 
CAR is expressed at very low levels in the normal adult heart, but it is highly expressed during cardiomyopathy or in a damaged heart. Therefore, CVB3 might potentially be useful as a vector that would allow the expression of exogenous proteins in the heart. Our previous studies have shown that replication-competent CVB3 vectors can be generated and used to facilitate gene transfer into neonatal and adult myocytes in culture and into adult mouse hearts in vivo [4]. However, this recombinant CVB3 vector elicits an immune response and causes tissue toxicity, which is a crucial drawback for a viral vector system. Coxsackievirus is typically considered a lytic virus that activates a potent immune response in the heart [5-7], but the recombinant CVB3 induces sparse inflammation in the heart and little myocardial injury [4]. Therefore, we investigated whether a replication-defective recombinant CVB3 could be engineered to express exogenous proteins in cardiomyocytes without cytotoxicity. Previous studies have explored the use of modified polioviruses as recombinant vectors to deliver genes into cells for the purpose of vaccination. A recombinant poliovirus was constructed in which a reporter gene was substituted for the P1 capsid gene and the deleted capsid gene was expressed by vaccinia P1 virus for recombinant virus production [8-12]. We used similar strategies to produce the replication-defective rCVB3.

Our findings suggest that a replication-detective rCVB3 vector can be used to facilitate gene transfer into neonatal myocytes in vitro and adult mouse hearts in vivo without cytotoxicity. These $\mathrm{rCVB} 3$ vectors might prove potential therapeutic tools to deliver exogenous proteins for treating human cardiovascular diseases.

\section{Materials and Methods}

\section{Cell Culture}

Human embryonic kidney (Hek) 293 and HeLa cells were maintained in Dulbecco's modified Eagle's medium with $10 \% \mathrm{fe}$ tal bovine serum (FBS; Invitrogen Co., Carlsbad, Calif., USA) and Chinese hamster ovary $(\mathrm{CHO})$ cells were maintained in RPMI 1640 (Invitrogen) with 10\% FBS, under standard cell culture conditions at $37^{\circ}$ and $5 \% \mathrm{CO}_{2}$ in air. Hek 293 cells were used for virus production because of their good transfection efficiency. Primary rat neonatal cardiomyocytes were isolated from the hearts of neonatal Sprague-Dawley rats less than 3 days old, and cultured as described [4].

\section{Cloning Recombinant Viral cDNA and Capsid Protein}

Expression Vectors

To test the application of $\mathrm{rCVB} 3$ as a nontoxic cardiotropic gene transfer vector, we used as the starting point GFP-CVB3 cDNA in pBluescript, as described previously [4], which con- tains the entire full-length CVB3 infectious cDNA and the sequence of an enhanced reporter gene for green fluorescent protein (GFP). The GFP-CVB3 sequence includes an in-frame synthetic polylinker containing the EcoRI and XhoI sites into which is inserted the GFP cDNA. The first six amino acids (MAAQEF) of the CVB3 VP4 capsid protein were inserted at the amino terminus of the GFP sequence. The luciferase ( $\mathrm{Lu}$ ) sequence was amplified by polymerase chain reaction (PCR) from the pGL3Basic plasmid (Promega, Madison, Wisc., USA), and used to substitute the GFP-P1 capsid protein coding sequences in GFPCVB3 [7] (pLuCVB3) (fig. 1A). For producing recombinant CVB3, the deleted CVB3 capsid P1 sequence was amplified by PCR and subcloned into the mammalian expression plasmid pcDNA3.1 (Invitrogen) using unique restriction enzyme sites (pCMV-P1) (fig. 1B). We used techniques similar to those that have been used to express exogenous antigens in a poliovirus vector system $[12,13]$.

Replication-Defective Recombinant LuCVB3 (rLuCVB3)

To generate the rLuCVB3 viral vector, the pLuCVB3 and pCMV-P1 plasmids were amplified and cotransfected into Hek293 cells using FuGENE 6 (Roche, Mannheim, Germany). The rLuCVB3 was recovered from the cell and culture supernatants for 3 days after transfection. The cells were broken down by double freeze/thaw cycle with subsequent centrifugation at $9,300 \mathrm{~g}$ for $30 \mathrm{~min}$ and filtration through a $0.22-\mu \mathrm{M}$ syringe filter [14]. The cleared extract was collected and stored at $-70^{\circ}$ before use. Generated rLuCVB3 was confirmed by luciferase activity in infected Hek293 cells. Hek293 cells were seeded at $10^{3}$ cells/well in 96-well plates and cultured for $24 \mathrm{~h}$; then rLuCVB3 supernatant was added to the wells and incubated for $24 \mathrm{~h}$ more. Luciferase expression was estimated using a firefly luciferase assay kit (Promega) and a single-tube TD20/20 luminometer (Turner Designs, Sunnyvale, Calif., USA). A cutoff point of 10 -fold induction of chemiluminescence relative to that of the uninfected control cells (set at 1) after normalization to the fluorescence of the uninfected control proteins (RLU/mg) was used to determine if the cells in a well had been infected successfully.

\section{Concentration of $r L u C V B 3$}

The rLuCVB3 virus vector was concentrated using a sucrose cushion method [15]. Cotransfection supernatants were centrifuged in a Beckman ultracentrifuge (XL-90 with an SW28 rotor) at $82,740 \mathrm{~g}$ for $30 \mathrm{~min}$ at $8^{\circ}$. The resulting supernatant was layered onto a $30 \%$ (w/v) sucrose cushion (in $10 \mathrm{mM}$ Tris pH 7.5, $100 \mathrm{mM}$ $\mathrm{NaCl}$ ) and ultracentrifuged using a SW-28 swing rotor for $18 \mathrm{~h}$ at $141,000 \mathrm{~g}$ at $8^{\circ}$ to pellet the viral particles. The rLuCVB3 pellet was resuspended in phosphate-buffered saline and stored at $-70^{\circ}$.

Estimation of the Titer of the Replication-Defective rLuCVB3

The rLuCVB3 vector has the capacity to infect cells but it cannot replicate and form plaques, so the viral titers cannot be quantified by traditional plaque-forming unit (PFU) assays. To overcome this problem, we used immunofluorescent staining for luciferase to estimate the titer of rLuCVB3 by comparison with the known titer of wild-type CVB3 [12]. The resulting titer was then expressed as infectious units. We determined that the infectious unit value of the replication-defective virus correlated significantly with the PFU of wild-type coxsackievirus. 


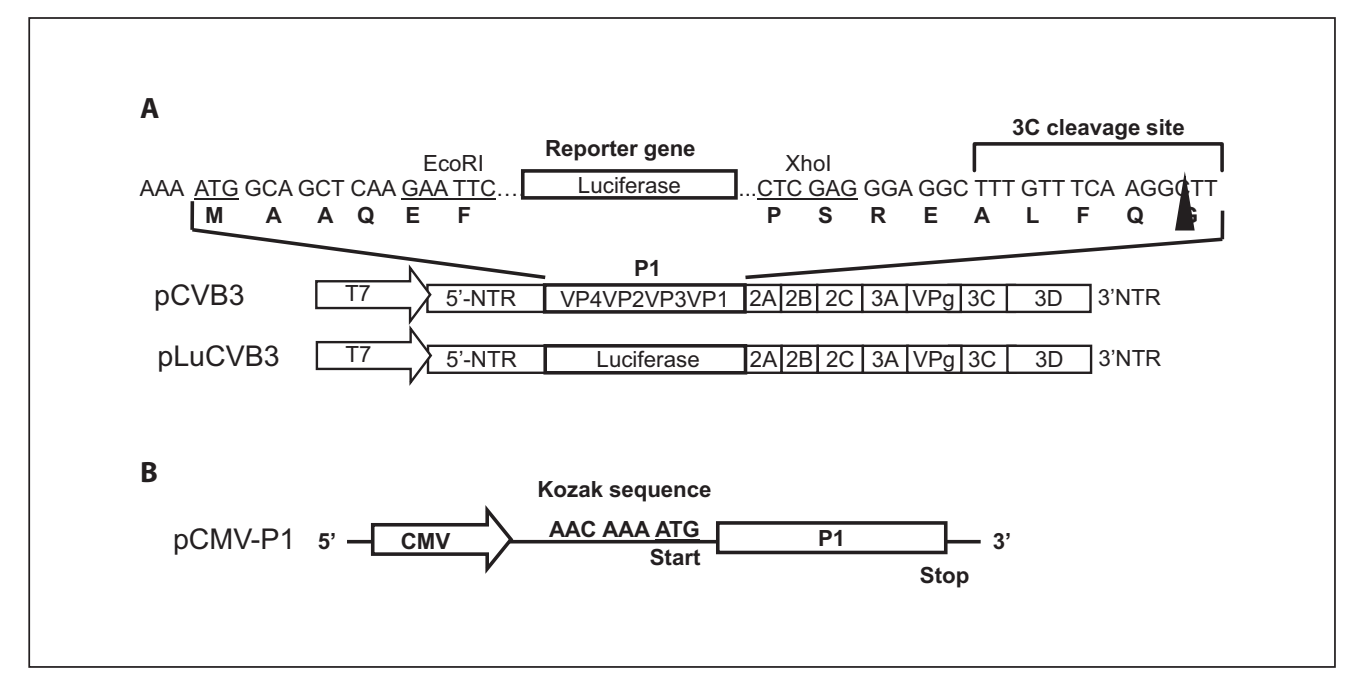

Fig. 1. A Schematics of capsid protein deleted replication-defective recombinant coxsackievirus cDNA and strategy for expression of the reporter gene. The black box at the $5^{\prime}$-end of the open reading frame indicates the exogenous gene (luciferase). The additional sequences in front of exogenous sequences are indicated: the start codon $(\mathrm{M})$ and additional amino acids (AAQEF) for a restriction enzyme polylinker. Additional sequences at the back of exogenous sequences are indicated: the polylinker site, the $3 \mathrm{C}^{\text {pro }}$ artificial

\section{Target Cell Infection and Measurement of Cytotoxicity}

Hek293, HeLa, CHO cells and cardiomyocytes ( $10^{3}$ cells) were seeded into the wells of 96-well plates using the appropriate medium as described above and cultured for $24 \mathrm{~h}$. rLuCVB3 was added into the wells at a multiplicity of infection (MOI) of 10 and incubated for a further $1 \mathrm{~h}$, after which the medium was changed to medium containing $1 \%$ FBS and the cells were cultured until assayed for luciferase activity and cytotoxicity. At 0, 2, 4, 8, 16, 24, 48 and $72 \mathrm{~h}$ postinfection (pi), the culture media were collected for the measurement of cytotoxicity using lactate dehydrogenase (LDH) assay kits (Roche). The cells were lysed directly in the culture wells and assayed with luciferase assay kits (Promega) according to the manufacturer's instructions.

\section{Western Blot Analysis}

Each cell was lysed in sodium dodecyl sulfate (SDS) sample buffer (25 mM Tris-HCl pH 6.8, 2\% w/v SDS, 10\% glycerol, $50 \mathrm{~mm}$ dithiothreitol and $0.1 \% \mathrm{w} / \mathrm{v}$ bromophenol blue). Aliquots of $10 \mu \mathrm{g}$ of total cell extracts were loaded onto $10 \%$ SDS-polyacrylamide electrophoresis (PAGE) gels and transferred to Hybond-ECL nitrocellulose membrane (Amersham, Bucks., UK). The membranes were blocked in 5\% nonfat dry milk solution (in Tris-buffered saline) containing $0.1 \%$ Tween-20. Protein bands were probed with anti-luciferase (NEB, Beverly, Calif., USA) and anti-tubulin (NEB) antibodies followed by labeling with a horseradish peroxidase-conjugated second antibody (Jackson, Baltimore, Md., USA). Bands were visualized using an enhanced chemiluminescence (ECL) kit (Amersham).

Foreign Gene Transfer to Cardiomyocyte without Cytotoxicity cleavage site (ALFQG), is indicated by a triangle. These recombinant CVB3 cDNAs were cloned into the pBluescript plasmid. B For encapsidation of the replication-defective $\mathrm{rCVB} 3$ vector, we generated a capsid protein expression plasmid (pCMV-P1). These plasmids have a complete open reading frame of $\mathrm{P} 1$ of $\mathrm{CVB} 3$ and the amino terminus was composed with optimized translation initiation Kozak sequences.

In vivo Analysis: $r L u C V B 3$ Delivers Reporter Protein to the Intact Myocardium in the Adult Mouse

The protocols used in this study conformed to the Guide for the Care and Use of Laboratory Animals published by the US National Institutes of Health (NIH Publ. No. 85-23, revised 1996). All procedures were reviewed and approved by the Institutional Animal Care and Use Committee of Samsung Biomedical Research Institute (SBRI). SBRI is accredited by the Association for Assessment and Accreditation of Laboratory Animal Care International (AAALAC International) and abides by the Institute of Laboratory Animal Resources (ILAR) guide. Six-week-old male $\mathrm{BALB} / \mathrm{c}$ mice were infected with $10^{5} \mathrm{PFU}$ rLuCVB3 through a tail vein injection. Mice were euthanized and the heart was collected at 1, 3 and 7 days pi to test luciferase expression and check on cardiac pathology. The basal parts of the hearts were homogenized in DMEM medium with 5\% fetal bovine serum and were detected luciferase expression using Western blot. The apical parts of the hearts were fixed in $10 \%$ formalin, embedded in paraffin wax, sectioned at $5 \mu \mathrm{m}$ and stained with hematoxylin and eosin (HE) and were detected luciferase expression and dystrophin disruptions using immunohistochemistry.

\section{Immunohistochemistry}

Immunohistochemical analysis of myocardial tissue sections was performed as described [16] using purified mouse anti-dystrophin IgG (Novocastra Laboratories Ltd, Newcastle upon Tyne, UK) and anti-luciferase (NEB). The avidin/biotin alkaline-phosphatase system (Vectorstain kit; Vector Laboratories, Burlingame, Calif., USA) was used for signal amplification. The slides 
Fig. 2. Cotransfection produced rLuCVB3 from Hek293 cells. A Cell supernatants were added into HeLa cell cultures to test cytotoxicity. After $48 \mathrm{~h}$, there were no significant changes in cell survival. B Detection of the reporter gene expression by luciferase activity. HeLa cells were incubated with different volumes of cell supernatants (virus volumes in $\mathrm{x}$-axis). Luciferase activity was increased dramatically in a volumedependent manner.

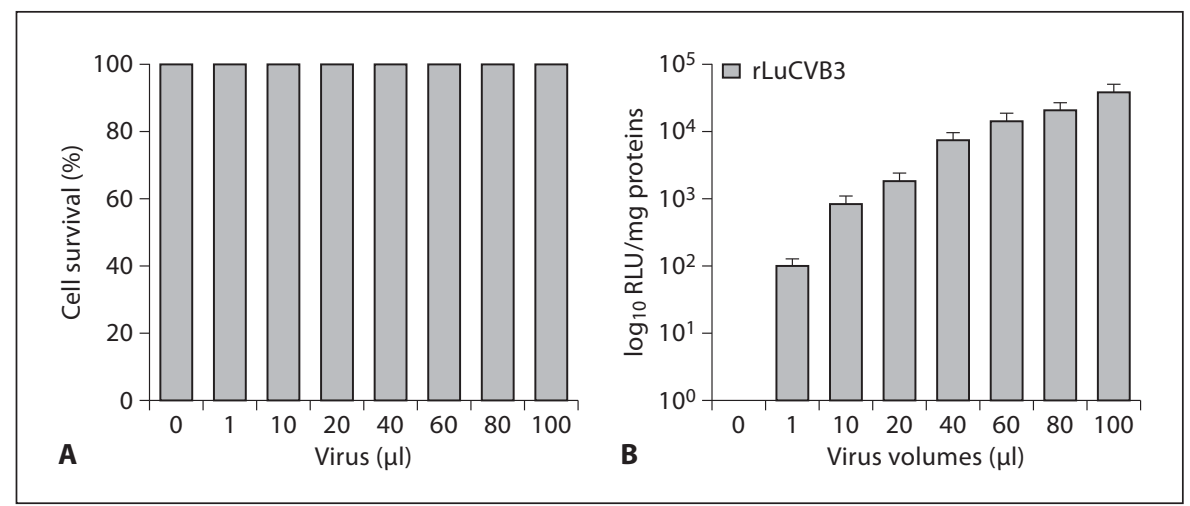

were sequentially treated with 3,3'-diaminobenzidine (DAB) Substrate Solution (Dako, Glostrup, Denmark) to develop the color reaction. Serial sections were stained with HE. Negative control staining was performed with blocking buffer instead of primary antibody.

\section{Statistical Analysis}

Data are presented as the mean \pm SEM. Student's t test was used for the analysis of numeric parameters (SPSS 10.0 for Windows; SPSS Inc., Chicago, Ill., USA). Differences were considered significant at $\mathrm{p}<0.05$.

\section{Results}

\section{Generation of Replication-Defective Coxsackievirus}

B3 Vector

To investigate nontoxic cardiac-specific gene delivery, we modified a CVB3 cDNA, which is a very well-defined highly cardiotropic enterovirus. To generate a replicationdefective CVB3, we cloned two different cDNA plasmids. One was a CVB3 backbone cDNA plasmid that replaced the capsid protein (P1: VP4, 2, 3 and 1) and carried a luciferase reporter gene for easy detection of virus gene delivery and for control of viral self-replication (pLuCVB3) (fig. 1A). The other was a virus capsid protein P1-producing plasmid with a mammalian CMV promoter to supply the deleted P1 protein (pCMV-P1) (fig. 1B). The infectious recombinant $\mathrm{CVB} 3$ virus (rLuCVB3) was recovered from cotransfection supernatants of both virus backbone and P1-expressing plasmids. After 3 days of cotransfection, Hek293 cells showed cytopathic effects indicating recombinant virus production.

\section{Recombinant CVB3 (rLuCVB3) Expresses Luciferase} in Infected Cells without Producing Cell Cytotoxicity

We examined rLuCVB3 infection and cell cytotoxicity on HeLa cell lines incubated with different volumes of
Table 1. Determination of the optimum structure for generating replication-defective rCVB3 virus after cotransfection of each capsid protein-deleted CVB3 cDNA with various capsid protein expression plasmids

\begin{tabular}{lcccc}
\hline rCVB3 cDNA & pCMV-VP0 & pCMV-VP3 & pCMV-VP1 & pCMV-P1 \\
\hline pLuCVB3 $\Delta$ VP0 & $<10^{2}$ & - & - & - \\
pLuCVB3 $\Delta$ VP3 & - & $<10$ & - & - \\
pLuCVB3 $\Delta$ VP1 & - & - & $<10$ & - \\
pLuCVB3 $\Delta$ P1 & - & - & - & $>10^{6}$
\end{tabular}

Results are represented as plaque-forming units $(\mathrm{PFU} / \mathrm{ml})$ and viral titers were determined by semiquantitation of luciferase immunofluorescent staining in HeLa cell monolayers. Values are means from independent experiments, in each of which titrations were carried out in triplicate.

rLuCVB3-containing cell supernatants. At $48 \mathrm{~h}$ after incubation, there was no decrease in cell viability compared with normal cell supernatants. Luciferase activity was also increased by the volume of cell supernatant. Aliquots of $1 \mu \mathrm{l}$ of cell supernatant produced $100 \mathrm{RLU} / \mathrm{mg}$ protein and $100 \mu \mathrm{l}$ resulted in 30,000 RLU/mg protein (fig. 2). These results suggest that $\mathrm{rLuCVB} 3$ could deliver the reporter gene to HeLa cells without causing cell cytotoxicity. The assumed presence of rLuCVB3 was also tested for its ability to affect cell proliferation. Fresh Hek293 cells were inoculated with supernatants that had been collected from Hek293 cell supernatants at $48 \mathrm{~h}$ pi with rLuCVB3. However, the luciferase activity in these cells remained negative, confirming the absence of progeny virus (data not shown).

We also studied the optimal structure of the capsid protein deletion for efficient production of rLuCVB3. It was confirmed by luciferase activity from HeLa 


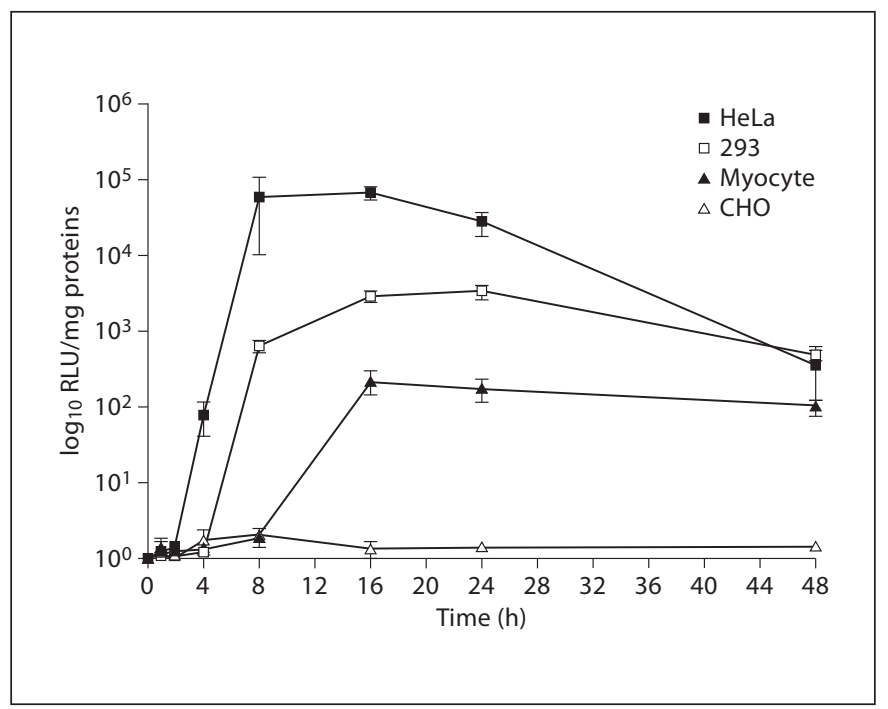

Fig. 3. Comparative infection of different cells with rLuCVB3 vector to investigate whether a replication-defective $\mathrm{rCVB} 3$ vectors could deliver foreign proteins into cells susceptible to wild-type coxsackievirus infections. Hek293T, HeLa, rat neonatal cardiomyocytes and $\mathrm{CHO}$ cells were infected with rLuCVB3 carrying genomes in which the $\mathrm{P} 1$ region was replaced by a luciferase reporter gene $(\mathrm{MOI}=10)$. Luciferase activity was measured at given time points postinfection. Representative data from four experiments are presented as the mean \pm SEM.

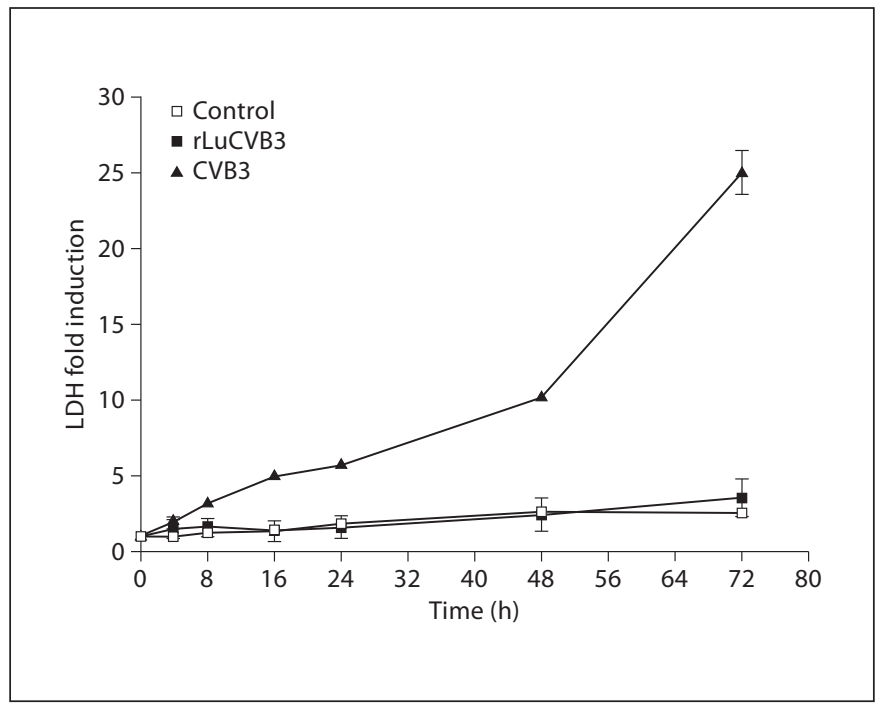

Fig. 4. Cytotoxicity of rLuCVB3 was tested on infected cardiomyocytes. The rLuCVB3-infected cell culture media supernatants were collected at given time points postinfection for measuring cytotoxicity using an assay of LDH activity. The LDH levels of wild-type CVB3 virus-infected cardiomyocytes increased at $16 \mathrm{~h}(4.94 \pm 0.03)$ and peaked at $72 \mathrm{~h}(24.82 \pm 0.04)$. However, the LDH level in rLuCVB3-infected cardiomyocytes was not significantly increased $(3.04 \pm 0.27)$, which was similar to the virus uninfected control cells $(2.8 \pm 0.02)$. Data are represented as means \pm SEM of the LDH fold changes from four experiment results. Each $\mathrm{LDH}$ fold change was compared with the $0 \mathrm{~h}$ baseline expressed as 1 .
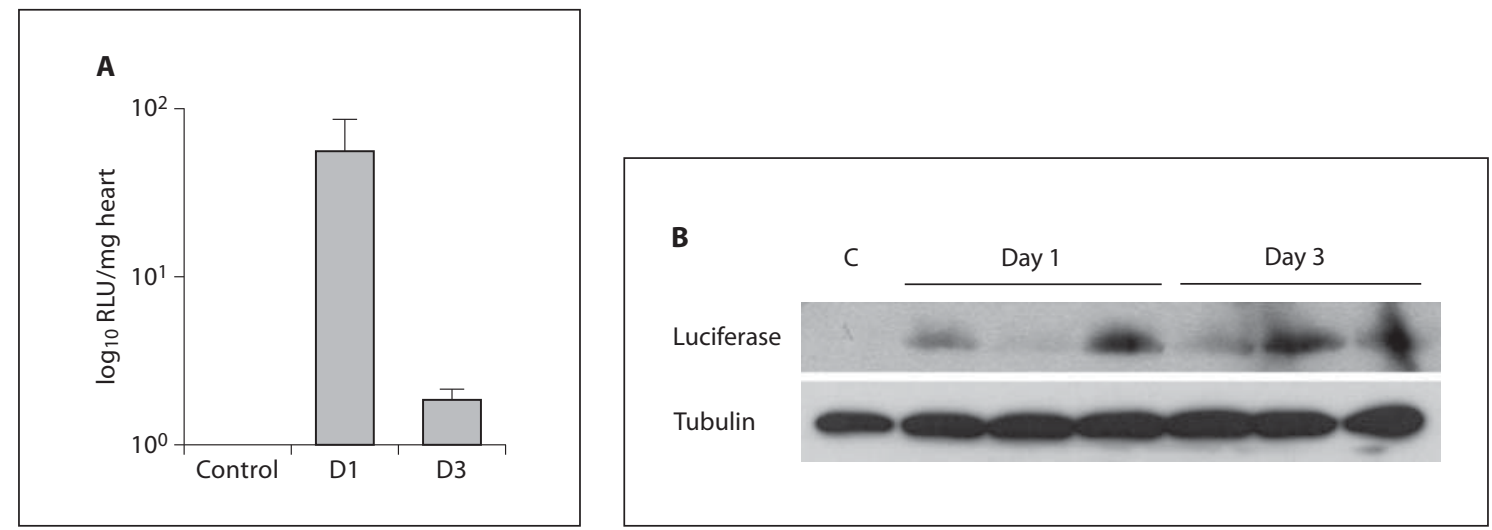

Fig. 5. Detection of foreign gene transfer in rLuCVB3-infected intact mouse hearts. Luciferase transfer was tested in the heart after a tail vein injection of 10 MOI of rLuCVB3. A Luciferase activity was detected from the hearts at days 1 and 3 ( $n=3$ for each time point). However, the enzyme activity was quickly eliminated after expression. B Protein was detected by Western blot analysis from three individual mice hearts. There were no significant decreases in the amount of luciferase protein detected. Tubulin was used as an internal control indicating the total protein amount in each sample. 
cells exposed to cotransfected cell supernatants. The pLuCVB3 $\Delta V P 0$, pLuCVB3 3 VP3 and pLuCVB3 3 VP1 structures did not produce significant amounts of recombinant CVB3. In contrast, the pLuCVB3 $\Delta \mathrm{P} 1$ (pLuCVB3) structure allowed the efficient production of rLuCVB3, with viral stocks of approximately $10^{6} \mathrm{PFU} / \mathrm{ml}$ obtained (table 1). We used this structure to generate rLuCVB3 in our subsequent experiments.

\section{rLuCVB3 Delivers Reporter Protein to Cultured Rat Neonatal Cardiomyocytes}

Next, we tested the infectivity of rLuCVB3 from various cell lines to investigate the delivery of a foreign protein to cells susceptible to wild-type CVB3. The susceptible cells - Hek293, HeLa and rat neonatal myocytes and unsusceptible $\mathrm{CHO}$ cells were infected with $10 \mathrm{MOI}$ of rLuCVB3. Cell lysates were then collected at 1, 2, 4, 8, 16,24 and $48 \mathrm{~h}$ pi. Luciferase activity was detected at $10^{5}$ $\log$ RLU/mg protein in Hek293 and HeLa cells and at $10^{2}$ $\log \mathrm{RLU} / \mathrm{mg}$ protein in cardiomyocytes at $16 \mathrm{~h}$ pi. All cell lines showed similar profiles of luciferase expression over $24 \mathrm{~h}$, although the expression level varied according to the cell infectivity. In particular, luciferase was constantly expressed over $48 \mathrm{~h}$ in cardiomyocytes. Luciferase activity was not detected in the $\mathrm{CHO}$ cells (fig. 3).

\section{rLuCVB3 Does Not Induce Cell Cytotoxicity in}

Cultured Neonatal Rat Cardiomyocytes

We found that rLuCVB3 could deliver a foreign protein to cardiomyocytes for $48 \mathrm{~h}$ and that there was no significant cytotoxicity in HeLa cells. Therefore, we investigated whether rLuCVB3 would induce cytotoxicity in cultured cardiomyocytes. To analyze the cytotoxicity of rLuCVB3, we measured LDH in the culture medium until $72 \mathrm{~h}$ pi. The LDH level in the culture medium of wild-type CVB3 increased gradually up to $72 \mathrm{~h}$ pi (30.8 \pm 0.04 -fold). However, rLuCVB3 produced no dramatic increase in $\mathrm{LDH}$ for this time $(2.5 \pm 0.27$-fold), similar to uninfected control cells ( $2.0 \pm 0.02$-fold) (fig. 4$)$. These results demonstrated that the recombinant CVB3 could deliver foreign proteins to cultured cardiomyocytes without cytotoxicity.

\section{rLuCVB3 Delivers Reporter Protein to the Intact Myocardium in the Adult Mouse}

To investigate whether rLuCVB3 delivers enough foreign protein to intact myocardium, mice were infected with $10^{5}$ PFU rLuCVB3 through a tail vein injection. Mice were euthanized and the heart was collected at 1 , 3 and 7 days pi to test luciferase expression and check on cardiac pathology. Luciferase activity was detected at
Fig. 6. $r L u C V B 3$ can deliver luciferase into the myocardium without inflammation. A Confirmation of rLuCVB3 gene transfer into the myocardium. The heart was harvested from a mouse at day 3 pi and luciferase was detected using immunohistochemistry. a Noninfected control, b rLuCVB3 infection; c and d show high magnifications of the rectangular area in the upper panel. Scale bar $=100 \mu \mathrm{m}$.
A
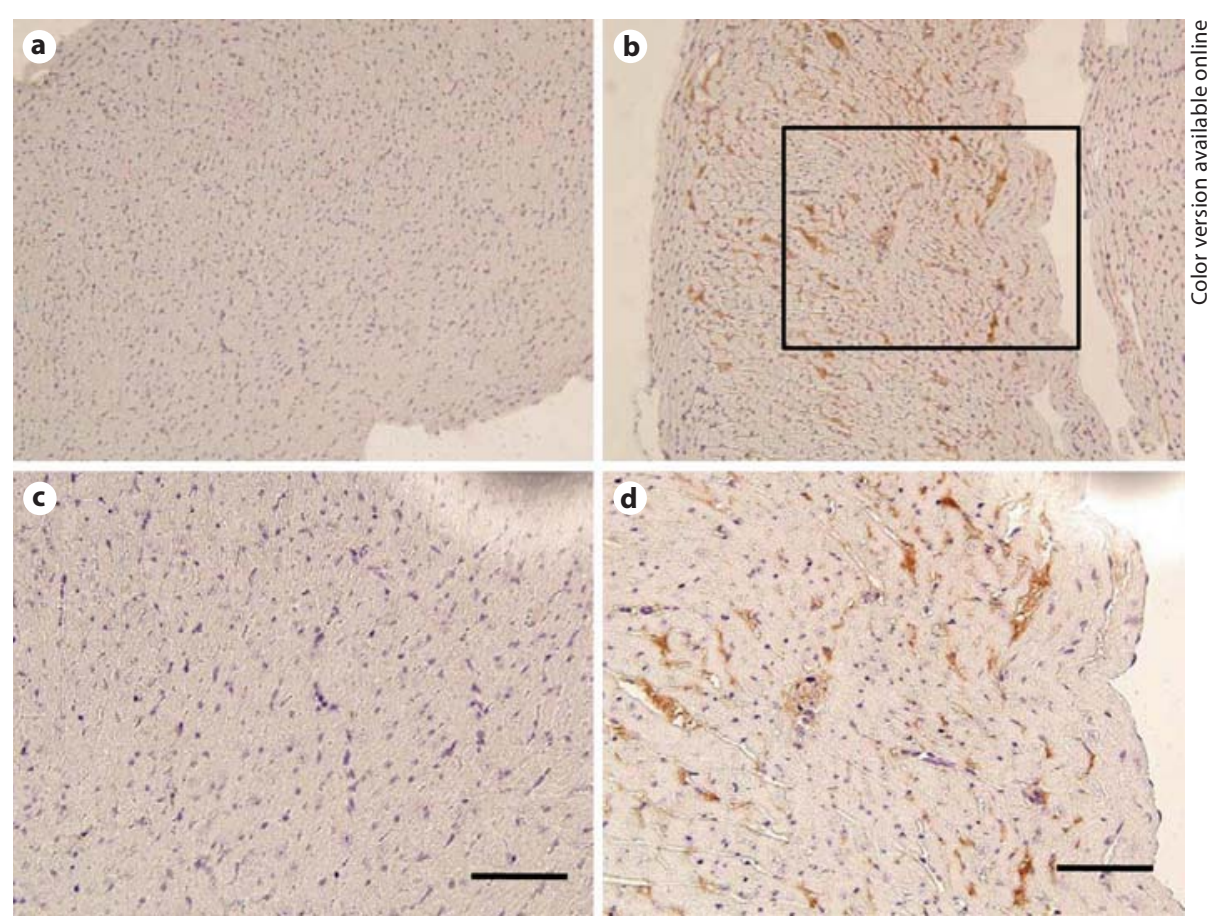
days 1 and 3 but was soon eliminated (fig. 5A). However, luciferase protein was detected at day 3 pi by Western blotting from three different hearts (fig. 5B). In addition, we examined the presence of luciferase in an individual myocardium. Luciferase was detected using immunohistochemistry in the myocardium at day $3 \mathrm{pi}$ (fig. 6A). Wild-type CVB3 is well known as a cause of viral myocarditis. Our results showed that $\mathrm{RLuCVB} 3$ infection did not induce inflammation in the mouse heart up to day 7 pi (fig. $6 \mathrm{Bb}, \mathrm{c}$ ), but CVB3 infection induced strong cardiomyocyte damage and inflammation in the heart (fig. 6Bd, e). In addition, the rLuCVB3-infected heart tissue did not exhibit dystrophin disruption (fig. 6Bg), as in control uninfected heart tissues (fig. 6Bf), but dystrophin disruption was noted following CVB3 infection (fig. 6Bh). These results demonstrated that rLuCVB3 could be used as a foreign protein delivery system into the heart without causing cardiomyocyte damage or inflammation.
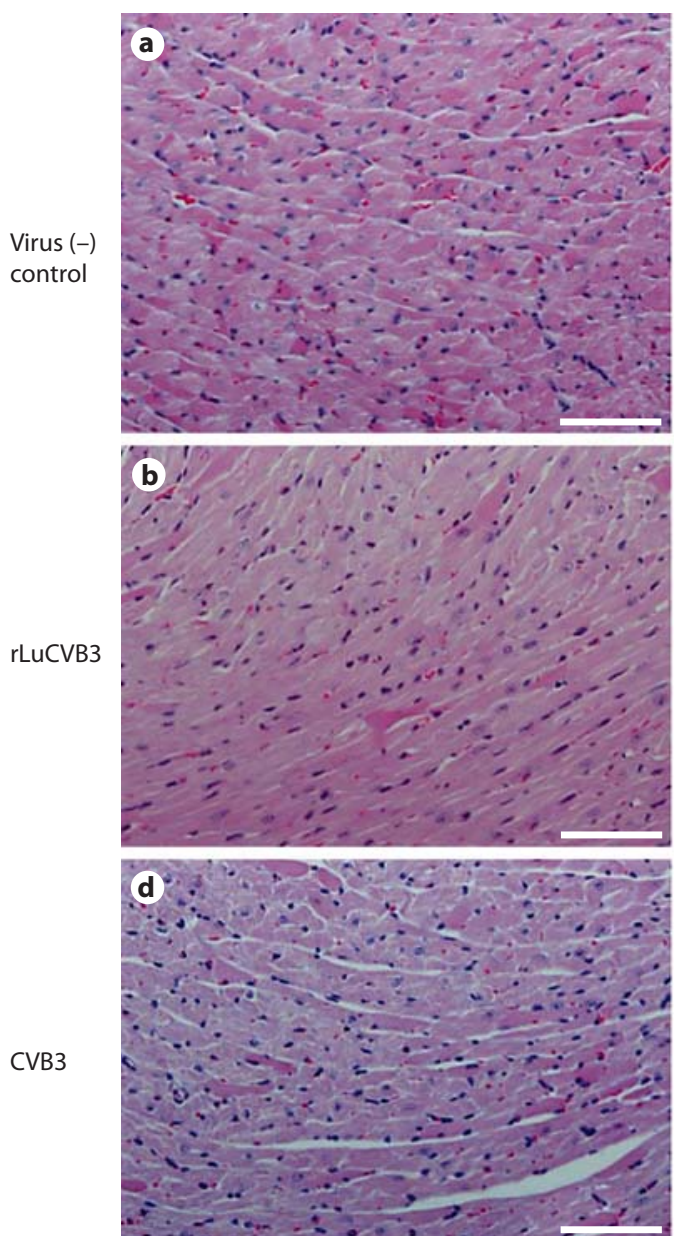

Day 3
$\mathrm{HE}$
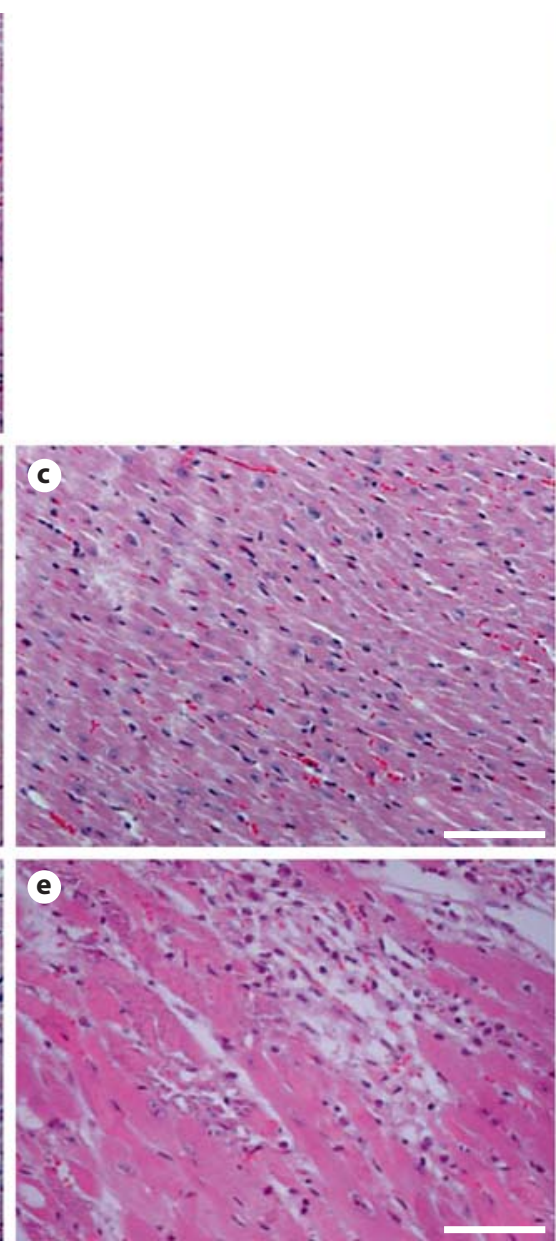

Dystrophin
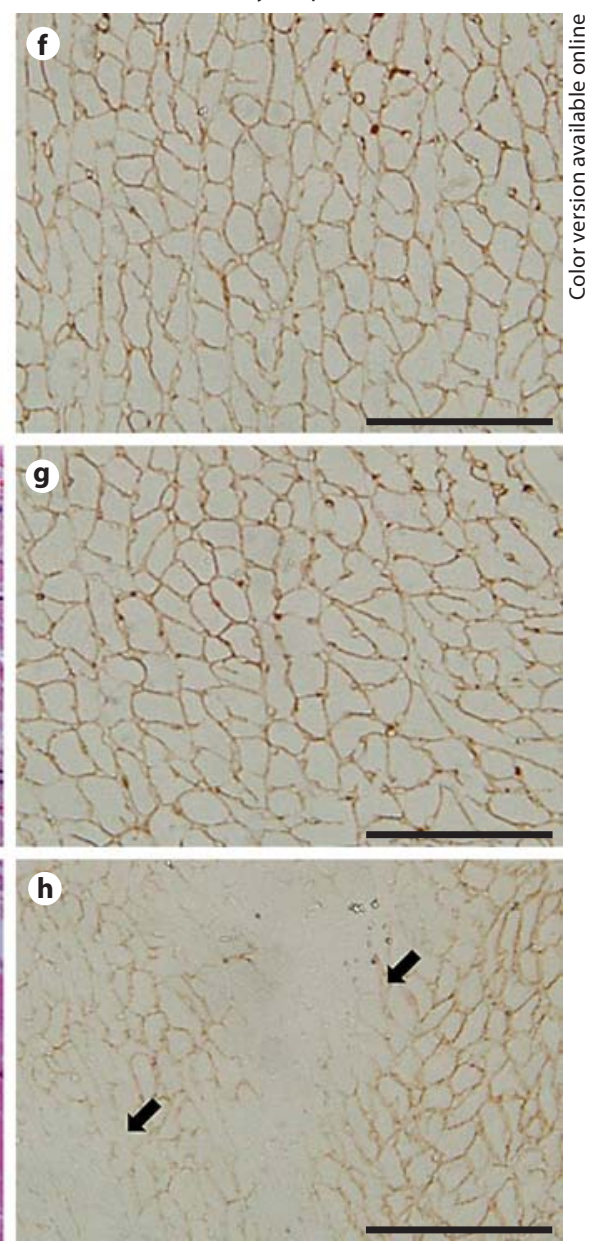

Day 7

Fig. 6. rLuCVB3 can deliver luciferase into the myocardium without inflammation. B rLuCVB3-induced cardiomyocyte damage was examined by histopathology with HE staining at days 3 and 7 pi. rLuCVB3 infection did not induce any severe inflammation or cell death $(\mathbf{b}, \mathbf{c})$. In contrast, wild-type CVB3 induced inflammation on days 3 and 7 pi (d, e). Dystrophin was not cleaved in the rLuCVB3-infected heart (g), but was cleaved in the CVB3-infected heart (h). Arrows indicate the dystrophin cleavage area (nonstained). Scale bar $=100 \mu \mathrm{m}$. 


\section{Discussion}

In this report, we have described a novel system for the generation of replication-defective rCVB3, which was developed for myocardial gene delivery without producing cytotoxicity. Previous experiments with poliovirus and CVB3 have demonstrated that picornaviruses can be used to express foreign polypeptides or antigens [13, 17-19]. We have also demonstrated that the coxsackievirus vector rCVB3-GFP can be used for long-term cardiac gene expression [4]. This rCVB3-GFP virus is a replication-competent virus vector and the immune response and tissue toxicity are crucial issues, as in any viral vector system. Although coxsackievirus is typically considered a lytic virus that activates a potent immune response in the heart [5-7], the rCVB3-GFP virus induced only sparse inflammation in the heart and little myocardial injury in a cardiac troponin $\mathrm{T}$ assay. The cytopathic effect is an important element in any viral vector system. Recent studies in our and other laboratories have suggested that a replication-defective coxsackievirus vector system could be used to deliver foreign proteins to cardiomyocytes without cytotoxicity [4]. In particular, studies on polioviruses have reported recombinant virus models [8-10, 12]. The relative tissue tropism of CVB3 is one of its significant differences from other viral vectors, such as adenoviruses, adeno-associated virus and retroviruses. Certain variants of CVB3, such as CVB3-H3, have been selected for their increased tropism for the heart by passage in mouse hearts $[7,20]$.

We generated three different systems, such as adenovirus vector, transgenic cell lines and mammalian expression plasmids, which expressed a single virus capsid protein such as VP0, VP3, VP1 or P1 region. Using cDNA in which each capsid protein region was deleted, we generated many different replication-defective rCVB3s. Initial tests showed that a recombinant $\mathrm{CVB} 3$ virus could be generated by the cotransfection of those plasmids: one allowing the transcription of the P1-deleted viral cDNA and the other permitting expression of $\mathrm{P} 1$ as a polyprotein. However, a single capsid protein deletion could not generate the rCVB3 virus. When the deleted capsid protein was supplied by an adenovirus vector (Ad-P1) or a transgenic cell line (293-P1) transfected with a replicon of the rCVB3 cDNA plasmid, there was no significant production of recombinant virus particles. Therefore, we used a plasmid cotransfection system to generate the replication-defective rCVB3 vector for further studies.

Previous studies have demonstrated that the trans encapsidation of a recombinant CVB3 virus was feasible using the cotransfection of a replicon plasmid, a plasmid expressing P1 capsid protein and a plasmid expressing $\mathrm{T}_{7}$ RNA polymerase. This produced titers of more than $10^{6}$ gene transfer units/ml with no further concentration steps [14]. However, these recombinant viruses had a cytopathic effect on the cells. One of the main advantages of the viral vector system presented here is that the replication-defective rCVB3 vector does not induce any cy topathic effect on the cells subjected to gene delivery.

In our experiments, we have shown that the replication-defective rCVB3 vector can be generated for use as a novel gene transfer vector to express exogenous genes in cardiomyocytes without cytotoxicity. The novel rCVB3 vector system is potentially a highly useful tool for delivering genes to cardiomyocytes. Further optimization of the protocol and improvement of the viral stock titers are the objectives of upcoming studies.

\section{Acknowledgment}

Supported by the Korea Research Foundation (KRF-2004041-E00092).

\section{References}

1 Wessely R, Henke A, Zell R, Kandolf R, Knowlton KU: Low-level expression of a mutant coxsackieviral CDNA induces a myocytopathic effect in culture: an approach to the study of enteroviral persistence in cardiac myocytes. Circulation 1998;98:450-457.

-2 Wessely R, Klingel K, Santana LF, Dalton N, Hongo M, Jonathan Lederer W, Kandolf R, Knowlton KU: Transgenic expression of replication-restricted enteroviral genomes in heart muscle induces defective excitationcontraction coupling and dilated cardiomyopathy. J Clin Invest 1998;102:1444-1453.
-3 Yu SF, Benton P, Bovee M, Sessions J, Lloyd RE: Defective RNA replication by poliovirus mutants deficient in 2 a protease cleavage activity. J Virol 1995;69:247-252.

$\checkmark 4$ Lim BK, Shin JO, Lee SC, Kim DK, Choi DJ, Choe SC, Knowlton KU, Jeon ES: Long-term cardiac gene expression using a coxsackieviral vector. J Mol Cell Cardiol 2005;38:745751.
5 Wessely R, Klingel K, Knowlton KU, Kandolf $\mathrm{R}$ : Cardioselective infection with coxsackievirus B3 requires intact type I interferon signaling: implications for mortality and early viral replication. Circulation 2001;103:756761.

6 Henke A, Nestler M, Strunze S, Saluz HP, Hortschansky P, Menzel B, Martin U, Zell R, Stelzner A, Munder T: The apoptotic capability of coxsackievirus B3 is influenced by the efficient interaction between the capsid protein VP2 and the proapoptotic host protein Siva. Virology 2001;289:15-22. 
7 Knowlton KU, Jeon ES, Berkley N, Wessely $\mathrm{R}$, Huber S: A mutation in the puff region of VP2 attenuates the myocarditic phenotype of an infectious CDNA of the Woodruff variant of coxsackievirus B3. J Virol 1996;70: 7811-7818.

$\checkmark 8$ Porter DC, Ansardi DC, Choi WS, Morrow $\mathrm{CD}$ : Encapsidation of genetically engineered poliovirus minireplicons which express human immunodeficiency virus type $1 \mathrm{Gag}$ and Pol proteins upon infection. J Virol 1993; 67:3712-3719.

$\checkmark 9$ Porter DC, Ansardi DC, Morrow CD: Encapsidation of poliovirus replicons encoding the complete human immunodeficiency virus type 1 Gag gene by using a complementation system which provides the P1 capsid protein in trans. J Virol 1995;69:1548-1555.

$\checkmark 10$ Porter DC, Ansardi DC, Wang J, McPherson S, Moldoveanu Z, Morrow CD: Demonstration of the specificity of poliovirus encapsidation using a novel replicon which encodes enzymatically active firefly luciferase. Virology $1998 ; 243: 1-11$.
11 Ansardi DC, Porter DC, Morrow CD: Complementation of a poliovirus defective genome by a recombinant vaccinia virus which provides poliovirus P1 capsid precursor in trans. J Virol 1993;67:3684-3690.

12 Ansardi DC, Moldoveanu Z, Porter DC, Walker DE, Conry RM, LoBuglio AF, McPherson S, Morrow CD: Characterization of poliovirus replicons encoding carcinoembryonic antigen. Cancer Res 1994;54: 6359-6364.

13 Andino R, Silvera D, Suggett SD, Achacoso PL, Miller CJ, Baltimore D, Feinberg MB: Engineering poliovirus as a vaccine vector for the expression of diverse antigens. Science 1994;265:1448-1451.

14 Meyer RG, Meyer-Ficca ML, Kaiser H, Selinka HC, Kandolf R, Kupper JH: Plasmidbased generation of recombinant coxsackievirus B3 particles carrying capsid gene replacement replicons. Virus Res 2004;104: 17-26.

15 Cunningham KA, Chapman NM, Carson SD: Caspase-3 activation and ERK phosphorylation during CVB3 infection of cells: influence of the coxsackievirus and adenovirus receptor and engineered variants. Virus Res 2003;92:179-186.
16 Oie E, Vinge LE, Tonnessen T, Grogaard HK, Kjekshus H, Christensen G, Smiseth OA, Attramadal H: Transient, isopeptide-specific induction of myocardial endothelin-1 MRNA in congestive heart failure in rats. Am J Physiol 1997;273:H1727-H1736.

17 Hofling K, Tracy S, Chapman N, Kim KS, Smith Leser J: Expression of an antigenic adenovirus epitope in a group B coxsackievirus. J Virol 2000;74:4570-4578.

$>18$ Slifka MK, Pagarigan R, Mena I, Feuer R, Whitton JL: Using recombinant coxsackievirus $\mathrm{B} 3$ to evaluate the induction and protective efficacy of CD8+ T cells during picornavirus infection. J Virol 2001;75:2377-2387.

19 Feuer R, Mena I, Pagarigan R, Slifka MK, Whitton JL: Cell cycle status affects coxsackievirus replication, persistence, and reactivation in vitro. J Virol 2002;76:44304440.

20 Van Houten N, Bouchard PE, Moraska A, Huber SA: Selection of an attenuated coxsackievirus B3 variant, using a monoclonal antibody reactive to myocyte antigen. J Virol 1991;65:1286-1290. 